\title{
3-D Integration Requirements for Hybrid Nanoscale-CMOS Fabrics
}

\author{
Pavan Panchapakeshan ${ }^{1}$, Priyamvada Vijayakumar ${ }^{1}$, Pritish Narayanan ${ }^{1}$, Chi On Chui $^{2}$, Israel Koren ${ }^{1}$ and \\ C.Andras Moritz ${ }^{1}$ \\ ${ }^{1}$ Department of Electrical and Computer Engineering, University of Massachusetts, Amherst, MA 01003, USA \\ ${ }^{2}$ Department of Electrical Engineering, University of University of California, Los Angeles, CA 90024, USA \\ Email:\{ppanchapakesh, andras\}@ecs.umass.edu
}

\begin{abstract}
Several nanoscale computational fabrics based on various physical phenomena have been proposed in recent years. However, their integration with CMOS has only received limited attention. In this paper we explore some of these integration challenges focusing on registration and the overlay between layers. We propose and evaluate a new 3D integration approach by carefully mixing standard CMOS design rules and nanoscale constraints. We address the following questions: (i) How much overlay precision is necessary? (ii) What is the impact on yield if different overlays are used?, and (iii) How can we mitigate the overlay requirements? For a nanoprocessor design implemented in $\mathbf{N}^{3}$ ASIC (a hybrid nanowire-CMOS fabric) we show that a $\mathbf{1 0 0 \%}$ yield is achievable even for a today's known overlay of $3 \sigma= \pm 8 \mathrm{~nm}$ (ITRS 2009). The $\mathbf{N}^{3}$ ASIC fabric version retains $6 \mathrm{X}$ density advantage compared to a projected 16nm CMOS scaled design even after 3D integration.
\end{abstract}

Index Terms - 3-D integration, Mask overlay, alignment.

\section{INTRODUCTION}

Manufacturing of integrated nano-systems with sublithographic structures continues to pose significant challenges. While unconventional manufacturing techniques such as imprint lithography [1] and SNAP [2] can produce ultra-dense regular structures at sub-10nm features; alignment with respect to previously formed patterns is still a concern (overlay alignment for imprint lithography is $3 \sigma$ $= \pm 105 \mathrm{~nm}$ [3]). Photolithography on the other hand has excellent mask overlay but may not achieve the same density. In this paper we propose a hybrid nano-CMOS 3-D integration approach that combines the advantages of unconventional and conventional manufacturing processes. We discuss the overlay requirements for hybrid nanofabrics, and show how full 3-D integration may be achieved using standard CMOS design rules. We discuss how design choices and order of process can mitigate overlay and alignment requirements, while retaining density benefits of sub-lithographic processes.

\section{3-D INTEGRATION REQUIREMENTS}

Nanofabrication techniques based on contact patterning or self-assembly based approaches tend to favor the formation of regular periodic structures such as grids. Registration requirements in such regular structures are alleviated since an initial lithography mask may be 'offset' with no loss of functionality. For example, NASICs [4][5] is a 2-D nanowire grid based fabric which uses lithography masks for functionalization, contacts etc. It was observed that a yield of $\sim 70 \%$ can be obtained for an overlay of $3 \sigma= \pm 5.7 \mathrm{~nm}$ (Manufacturing solutions known - ITRS[6]). In this paper, we discuss how regular nanofabrics could be built with full 3-D integration, while addressing overlay requirements and density implications.

One approach to build a fully integrated 3-D fabric is to use only optical lithography for all process steps. While the overlay precision is projected to be excellent and yield very high, this approach is expected to have low density when compared to techniques that use self-assembly/ unconventional nanofabrication techniques.

A second approach would be to combine unconventional and conventional manufacturing flow to obtain a 3D integrated fabric of high density. Such an approach has been adopted in CMOL [7] and HP's FPNI [8] nanofabric, where unconventional techniques such as nanoimprint are necessary after the fabrication of CMOS layers. As mentioned previously, overlay alignment for imprint lithography is $3 \sigma= \pm 105 \mathrm{~nm}$ [3], which implies significant challenges in alignment against previously formed features. Such a large overlay misalignment can contribute to significant yield loss (or conversely trading-off much of the density benefit for acceptable yield) and is not ideal.

Conventional CMOS manufacturing flow guarantees very high alignment and overlay precision but fails to realize a highly dense nanofabric. The unconventional nanomanufacturing techniques guarantee a highly dense fabric but have very poor alignment precision with respect to previously formed patterns. With this learning, we propose a nano-CMOS integration approach which considers the order of manufacturing process steps along with fabric design choices which aid in mitigating mask overlay while still achieving an ultra dense fabric.

It was seen that a uniform nanowire grid structure at the bottom implies that the first process step may be 'offset' with no loss of functionality. Furthermore, if an unconventional manufacturing step is performed before any lithographic masking, it is not affected by any overlay requirement. This fact motivates us to utilize the uniform nanowire grid as the bottommost layer in the 3-D integration approach. The regular structure mitigates the impact of mask offset while 


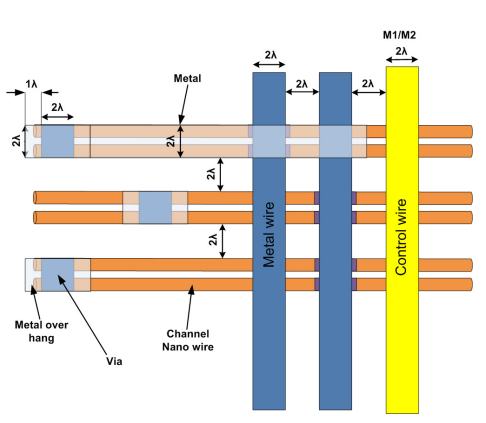

Fig.1 NW design rules for 3-D integration

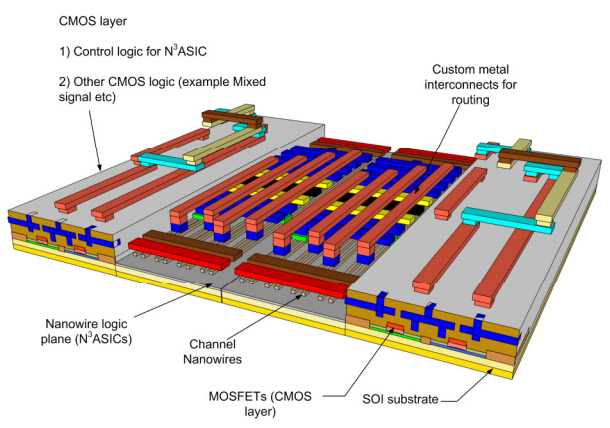

Fig. 2 A nano-CMOS 3D integrated fabric

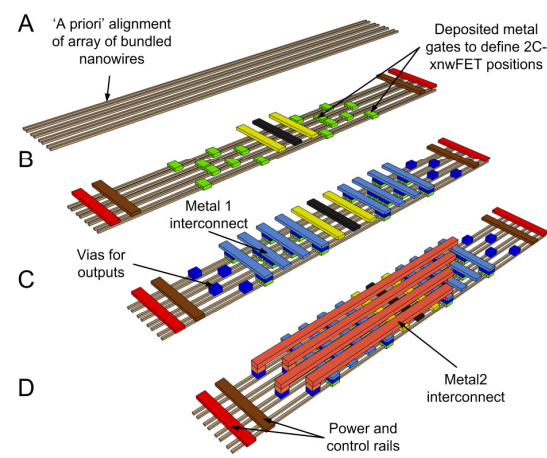

Fig. 3 A simplified manufacturing sequence overlay requirements are removed ensuring finer nanoscale resolution (and consequently higher density) than can be achieved with lithography at the bottom.

In order to successfully achieve 3-D integration with good overlay precision, conventional lithography is used and CMOS design rules (Fig. 1) are followed for all subsequent steps such as creation of metal vias, interconnect, contact rails etc. Fig. 1 also shows the design rules across nanoscale features and lithographic scale length $\lambda$, to accommodate via placement. Pitch and spacing dimensions of the bottom nanowire grid must adhere to CMOS design rules.

A fabric incorporating these principles of 3-D integration is Nanoscale 3-D Application Specific Integrated Circuits ( ${ }^{3} \mathrm{ASICs}$ )[9] (Fig. 2). The step-by-step 3-D integration approach for $\mathrm{N}^{3}$ ASICs is shown in Fig. 3. Nanowires may be direct-patterned on Silicon-on-Insulator substrates (Fig. 3A) through unconventional approaches such as SNAP and imprint lithography. Since metal vias are used as contacts for the channel nanowires, the spacing of the channel nanowires is determined by via spacing. Since channel nanowires could have much smaller dimensions than metal vias, they are bundled into pairs to make better contact, and provide for dual channel crossed-nanowire field-effect transistors (2C-xnwFETs) [9].

Following the a priori patterning of nanowire layers, masks are used for metal gate deposition. This step defines the positions of the transistors on the grid to achieve the required functionality (Fig. 3B). Finally, metal stacks implement interconnects as in traditional CMOS (Fig. 3C, 3D). The fabric can be built on a single SOI wafer, with nanowire logic plane surrounded by CMOS circuitry and is found to be $6 \mathrm{X}$ denser than CMOS.

\section{OVERLAY SIMULATION RESULTS}

The WISP-0 nanoscale processor design [4] was

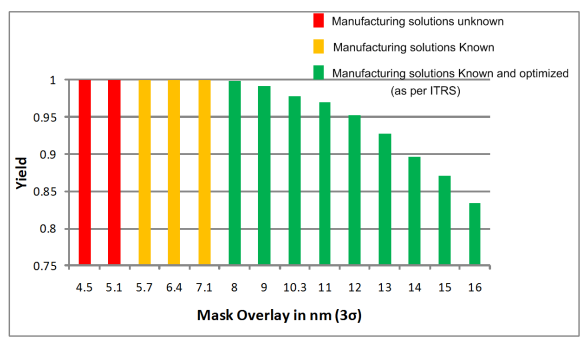

Fig. 4 Yield vs. Overlay for 3D integrated fabric mapped onto the $\mathrm{N}^{3} \mathrm{ASIC}$ fabric mentioned above. Overlay misalignments were modelled as Gaussian random variables, and Monte Carlo simulations were carried out in a detailed logic simulator to determine the number of functioning chips. The simulations were carried for the $3 \sigma$ overlay values projected by ITRS. The results in Fig. 4 show that close to $99 \%$ yield may be obtained for $3 \sigma= \pm 9 \mathrm{~nm}$ overlay (manufacturable as per ITRS) when constructing a uniform nanowire bundle with $\lambda=8 \mathrm{~nm}$ ( $16 \mathrm{~nm}$ technology node) in the 3D integrated fabric. Within a bundle widths of nanowires were $5 \mathrm{~nm}$, with $6 \mathrm{~nm}$ spacing to accommodate $16 \mathrm{~nm}$ vias. Fig. 4 shows that a pessimistic mask overlay with $3 \sigma= \pm 16 \mathrm{~nm}$ results in a yield of $83 \%$.

\section{CONCLUSION}

We show that the design choices and the order of process aid in mitigating the impact of mask overlay. The proposed $\mathrm{N}^{3} \mathrm{ASIC}$ fabric defined by a dense nanowire array at the bottom, followed by CMOS interconnect layers on the top is $6 \mathrm{X}$ denser than CMOS and is realizable with the available manufacturing techniques at very minimal yield loss. Any overlay precision better than $9 \mathrm{~nm}$ results in a yield of $100 \%$. In contrast, irregular structures would have more stringent mask overlay requirements.

\section{REFERENCES}

[1] T. Mårtensson, et al. , "Nanowire Arrays Defined by Nanoimprint Lithography," Nano Letters, vol. 4, no. 4, pp. 699-702, Apr. 2004.

[2] D. Wang, et al., "Two-dimensional single-crystal nanowire arrays," Small, vol. 3, pp. 2043-2047, 2008

[3] C. Picciotto, J. Gao, Z. Yu, and W. Wu, "Alignment for imprint lithography using nDSE and shallow molds," Nanotechnology, vol. 20, no. 25, p. 255304, 2009.

[4] C. A. Moritz, P. Narayanan, and C. O. Chui, "Nanoscale Application Specific Integrated Circuits," Book Chapter for Nanoelectronic Circuit Design (Ed. Niraj Jha), to appear 2011

[5] Teng Wang, P. Narayanan, M. Leuchtenburg, and C. Moritz, "NASICs: A nanoscale fabric for nanoscale microprocessors," in Nanoelectronics Conference, 2008. INEC 2008. 2nd IEEE International, pp. 989-994, 2008.

[6] "ITRS Home." [Online]. Available: http://www.itrs.net/.

[7] K. K. Likharev, "CMOL: Second life for silicon?," Microelectronics Journal, vol. 39, pp. 177-183, Feb. 2008.

[8] G. S. Snider and R. S. Williams, "Nano/CMOS architectures using a field-programmable nanowire interconnect," Nanotechnology, vol. 18 , no. 3, p. 035204, 2007.

[9] P. Panchapakeshan, P. Narayanan, and C. A. Moritz, "N3ASICs: Nanoscale 3-Dimensional Application Specific Integrated Circuits," to be submitted to NANOARCH 2011. 